\title{
Reinserción social de adolescentes en conflicto con la ley. \\ Un estudio conceptual
}

Social Reintegration of Juveniles in Conflict with the Law.

A Conceptual Study

Reinserção social de adolescentes en conflito com a lei. Un estudo conceitual

\author{
LizeTH MONTEJANO TORRES* \\ Jaime Sebastián F. GaLÁn JimÉnEZ ** \\ PaOla Iliana de La Rosa RodRíGUeZ ${ }^{\star \star *}$
}

FECHA DE RECEPCIÓN: 28 DE ENERO DE 2019. FeCHA DE APROBACIÓN: 30 DE ABRIL DE 2019

Doi: http://dx.doi.org/ 10.12804/revistas.urosario.edu.co/sociojuridicos/a.7606

Para citar: Montejano Torres, L., Galán Jiménez, J. S., \& De la Rosa Rodríguez, P. I. (2019). Reinserción social de adolescentes en conflicto con la ley. Un estudio conceptual. Estudios Socio-jurídicos, 22(1), 233-262. Doi: http://dx.doi.org/ 10.12804/revistas.urosario.edu.co/sociojuridicos/a.7606

\section{RESUMEN}

El objetivo de esta investigación consiste en hacer una revisión sobre las medidas y formas en que se ha empleado el concepto de reinserción social adolescente. Desde diversas disciplinas y sectores sociales en México, ha comenzado el reconocimiento de las personas jóvenes como un grupo de importancia que debe ser atendido dentro de las políticas públicas; sin embargo, quienes se encuentran en conflicto con la ley rara vez son considerados en los programas gubernamentales. Desde la perspectiva psicojurídica podría darse una aproximación a partir de la teoría del aprendizaje social y el etiquetamiento.

* Maestra en Derechos Humanos y Licenciada en Psicología de la Universidad Autónoma de San Luis Potosí, México. Correo electrónico: psic.montejano@gmail.com. ORCID: https:// orcid.org/0000-0002-8325-983X.

** Doctor, maestro y licenciado en psicología. Candidato al Sistema Nacional de Investigadores. Docente investigador de la Universidad Autónoma de San Luis Potosí y coordinador del centro de orientación psicológica. Correo electrónico: Sebastian.fgalan@uaslp.mx psic.sebastiangalan@gmail.com. ORCID: http://orcid.org/0000-0002-8801-5201

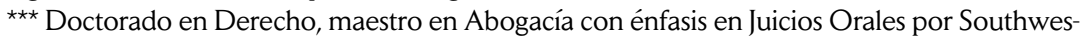
tern Law School. Maestro en Derecho Internacional por ITESM, licenciado en Derecho de la Universidad Autónoma de San Luis Potosí. Profesor-investigador de la UASLP, miembro del Sistema Nacional de Investigadores nivel 1. Correo electrónico: paola.delarosa@uaslp.mx. ORCID: http://orcid.org/0000-0001-6620-3589 
Método: se realizó una revisión sistemática de literatura científica publicada en el periodo comprendido entre enero de 2012 y junio de 2017, utilizando la herramienta de Google Académico con el concepto de reinserción social. La muestra total fue de 93 artículos de los cuales se retuvieron sólo 18 que cumplían con los criterios de inclusión. Los resultados sugieren que la reinserción social de personas adolescentes no difiere del que se utiliza para población adulta.

Palabras clave: reinserción social, adolescentes en conflicto con la ley, privación de la libertad, aprendizaje social.

\title{
ABSTRACT
}

This article aims to analyze the different approaches given to the concept of social reintegration as well as to its applications in teenagers. With different perspectives, Mexico is beginning to look at youngsters as an essential group. In fact, it has recognized that public policies should be involved when targeting this group. However, government programs rarely consider those in conflict with the law. From the psycho-juridical view, the phenomenon could be studied from the social learning theory and social labeling. The method used was a systematic review of the social reintegration concept within scientific literature in the period from January 2017 to June 2017 using Google Scholar. Out of 93 articles obtained, 18 met the criteria and were used in the study. Results suggest that social reintegration in teenagers does not differ from the concept used when applied in adults.

Keywords: Social reintegration, adolescents in conflict with the law, deprivation of liberty, social learning.

\section{RESUMO}

O objetivo desta pesquisa consiste em fazer uma revisão sobre as medidas e formas em que se tem empregado o conceito de reinserção social adolescente. Desde diversas disciplinas e setores sociais no México, tem começado o reconhecimento das pessoas jovens como um grupo de importância que deve ser atendido dentro das políticas públicas; no entanto, quem encontram-se em conflito com a lei, rara vez, são considerados nos programas governamentais. Desde a perspectiva da psicologia jurídica poderia se dar uma aproximação a partir da teoria da aprendizagem social e a rotulagem. Método: se realizou uma revisão sistemática de literatura científica publicada no período de janeiro de 2012 até junho 2017 empregando a ferramenta de Google Acadêmico com o conceito de reinserção social. A amostra total foi de 93 artigos, dos quais se retiveram só 18 artigos que cumpriram com os critérios de inclusão. Os resultados sugerem que a reinserção social de pessoas adolescentes, não diferem do que se utiliza para a população adulta.

Palavras-chave: reinserção social, Adolescentes em conflito com a lei, Privação da liberdade, aprendizagem social.

\section{Introducción}

\author{
De acuerdo con la Encuesta Nacional de Victimización y Percepción \\ sobre Seguridad Pública del Instituto Nacional de Estadística y Geografía \\ (INEGI, 2018), la tasa de incidencia delictiva en México hasta 2017 fue
}


de 39.369 por cada cien mil habitantes. Entre los delitos del fuero común más usuales de los que la ciudadanía reportó haber sido víctima se encontraron: el robo o asalto en la calle o transporte público, la extorsión, el fraude, el robo total y parcial de vehículos, las amenazas verbales, el robo en casa, habitación y lesiones. Según el INEGI (2017) en 2016, se encontraban 1913 personas adolescentes en situación de internamiento, de las cuales $71 \%$ corresponden a la comisión de una conducta tipificada como delito del fuero común y $29 \%$ del fuero federal. Así mismo, se reportó el ingreso de 4.507 adolescentes (7,07\% mujeres y $92,92 \%$ hombres) y el egreso de 6.137 adolescentes (6,95\% mujeres y 93,04\% hombres) de alguno de los 55 centros especializados del país. Por otra parte, se encontraban 6103 adolescentes $(6,60 \%$ mujeres y $93,39 \%$ hombres) en tratamiento externo.

En 2006 se evidenció un aumento en la participación de menores de edad en actos delictivos (Red por los derechos de la infancia, 2011), hecho relacionado estrechamente con el periodo en que Felipe Calderón lanzó una campaña contra el narcotráfico, también conocida como "Guerra contra el narcotráfico" (Rosen Zepeda, 2015). Esta acción dio inicio a uno de los periodos más violentos que hasta entonces había atravesado México (Daniel \& Zepeda, 2015; Aguilar \& Castañeda, 2012), pues el número de homicidios se incrementó un 24\%, hasta llegar a 27.213 casos solo en el 2011 (Molzahn, Rodriguez \& Shirk, 2013).

Durante los años comprendidos entre 2007 y 2010, de acuerdo con el "Informe alternativo sobre el Protocolo Facultativo de la Convención sobre los derechos del niño relativo a la participación de niños en los conflictos armados" (Red por los derechos de la infancia, 2011), fueron detenidos 232 menores de edad por la Secretaría de la Defensa Nacional, 26 por la Secretaría de Marina y 363 por la Policía Federal, en operativos militares contra la delincuencia organizada. Además, la Procuraduría General de la República registró la detención de al menos 3664 menores en operativos federales contra la delincuencia organizada desde diciembre de 2006 hasta abril de 2010.

Estos acontecimientos pudieron generar un estigma hacia determinados jóvenes, quienes al poseer características específicas de marginación económica, social, urbana, laboral, educativa y cultural semejantes o compartidas con jóvenes que participaron en actividades delictivas, se 
han convertido en objeto de atención y vigilancia particular, aún y cuando no se les ha comprobado la realización de algún acto delictivo; constituyendo con esto lo que algunos autores identifican como criminalización de la juventud (Valenzuela, 2015; di Napoli, 2016).

Lo anterior se relaciona con la teoría del etiquetamiento social o labelling approach desarrollada por Becker (1963), en la que describe que una conducta se considera como desviada cuando el colectivo social la califica con esa etiqueta, consecuencia de la aplicación de registros y sanciones estipulados, siendo así que el desviado (en este caso el joven delincuente u ofensor) será aquel que ha sido etiquetado como tal. Una cuestión importante de esta propuesta radica en que un comportamiento puede ser etiquetado o identificado como criminal, sin que sea constitutivo de un delito; por lo tanto, un comportamiento desviado es aquel que se opone o enfrenta a la norma y que es percibido de esa forma.

Es debido a esto que es crucial la tipificación expuesta en el cuerpo legislativo, pues es en este donde queda explícita la acción que el Estado deberá de ejercer. Lo anterior queda sujeto al contexto, la circunstancia y el ejecutor, pues estos datos determinan que una acción sea catalogada como delincuencial o no (Pérez, 2011); por ejemplo, la acción de privar a una persona de la vida es tipificada como homicidio en las legislaciones penales y es un acto condenado por la sociedad; sin embargo, el tiempo de la medida que le será impuesta por parte de la autoridad penal dependerá de las circunstancias en las que ocurrió el hecho, si es mayor o menor de edad quien lo realizó, así como el tratamiento al cual quedará sujeto dentro de la institución de seguridad en la que sea recluido.

Con relación a este tema, en México, el 18 de junio de 2008 se realizó una reforma a la Constitución Política de los Estados Unidos Mexicanos en materia penal y seguridad pública (CPEUM, art. 18, 2008), la cual reveló la transición hacia un sistema acusatorio respondiendo con esto a "la necesidad que existía de ampliar el ámbito de protección de Derechos Humanos en materia procesal penal" (Mendoza, 2011, p. 16). En esta modificación quedó asentado que la organización del sistema penitenciario tiene como base cinco ejes principales que son: el trabajo, la capacitación en dicho sentido, la educación, la salud y el deporte, los cuales se pretende que sirvan como medios para lograr que la persona sentenciada logre la reinserción en la sociedad, procurando siempre que 
no vuelva a delinquir (CPEUM, 2018, art. 18), cabe señalar que ésta es la primera vez que se hace uso del término "reinserción social" en el contenido de la Carta Magna.

En el caso particular de la justicia de menores de edad, en 2016 se establece el Sistema Integral de Justicia para Adolescentes en la República Mexicana como resultado de la expedición de la Ley Nacional del Sistema Integral de Justicia Penal para Adolescentes (LNSIJPA, 2016). En este modelo integral, las medidas de sanción poseen un carácter socioeducativo y no punitivo, con las cuales se busca insertar a la persona adolescente en su familia y en la sociedad, mediante el desarrollo de sus capacidades y sentido de responsabilidad (LNSIJPA, 2016, art. 30, párr. 2).

Para los adolescentes cuyo proceso legal culmina con la imposición de una medida de internamiento, la LNSIJPA (2016) expone que esta será usada como última opción, y por el tiempo más breve que proceda, de acuerdo al grupo etario al que pertenezcan (art. 164, párr. 1), por lo que en el caso de adolescentes entre 14 años a 16 años (grupo etario II), correspondería una duración máxima de tres años (art. 145, párr. 4), y quienes tengan de 16 a 18 años (grupo etario III), aplicarían un máximo de cinco años (art. 145, párr. 5).

Dentro de la LNSIJPA (2016) se define la reinserción social como "la restitución de pleno ejercicio de los derechos y libertades tras el cumplimiento de las medidas ejecutadas" (art. 29); sin embargo, no va más allá del alcance jurídico y no indica los parámetros que estimarían que un adolescente ha logrado este objetivo. Cabe señalar los requerimientos establecidos por los ordenamientos jurídicos internacionales aplicables a menores de edad en conflicto con la ley en México, algunos de los cuales se enuncian a continuación, ya que brindan una aproximación a lo que es la reinserción social de personas privadas de su libertad y/o dirigidas hacia adolescentes:

a) Pacto Internacional de Derechos Civiles y Políticos (1966), en este instrumento, el fin del régimen penitenciario es reformar y readaptar a los penados.

b) Las Reglas mínimas de las Naciones Unidas para la Administración de la Justicia de Menores, conocidas también como "Reglas de Beijín" (1985), considera la capacitación y el tratamiento de menores con el objetivo de que desempeñen un papel productivo en la sociedad. 
c) Las Reglas de las Naciones Unidas para la protección de los menores privados de libertad o "Reglas de la Habana" (1990) habla de beneficiarlos con las medidas que puedan ser concebidas para ayudar a reintegrarles después de ser puestos en libertad, particularmente en los grupos de la comunidad, la familia y la educación; además, las actividades y programas a los que queden sujetos deben promover el sano desarrollo y su dignidad, cumpliendo con los derechos de recibir una enseñanza adecuada, formación para ejercer un oficio y en caso de ser posible, la oportunidad de realizar un trabajo remunerado.

d) La Convención sobre los Derechos del Niño (1990), considera el derecho del menor a mantener contacto con la familia por medio de correspondencia y visitas.

e) Los Principios básicos para el tratamiento de reclusos (1990) resalta la participación activa de la comunidad e instituciones sociales para que la persona recluida una vez terminando su sanción, logre reincorporarse a la sociedad de la mejor manera posible.

Si bien la reinserción social como concepto ha sido abordada desde diversas disciplinas, debido a la escasez en el desarrollo teórico, así como de evaluaciones sistemáticas que le den contenido, la definición aceptada dentro del ámbito jurídico penal suele tomar como base lo establecido por los estatutos legislativos adoptados por el Estado o, en su defecto, se utilizan de manera indiscriminada los conceptos como reintegración, readaptación y rehabilitación, evidenciando la visión que la legislación en turno tiene sobre las personas que delinquen, así como la forma en como se les valora socialmente (Villagra, 2015).

Lo anterior ejemplifica la crisis de los $r e^{1}$ de la que habla Zaffaroni (1995), para quién, en el fondo, los términos únicamente discriminan socialmente y sirven a los discursos en los ámbitos académicos y políticos, dejando de lado la aplicación real dentro de las instituciones, la posible 
utilidad para las personas que operan en las mismas y sobre todo, para quienes van dirigidas las acciones de manera directa por encontrarse cumpliendo con una medida de sanción.

Desde esta postura, es pertinente examinar el sistema de valores y los comportamientos imperantes que se presentan en la sociedad en la que se pretende reintegrar a las personas que se encuentran cumpliendo con alguna sanción penal (Baratta, 2004; Valenzuela, 2015). También se relaciona íntimamente con los análisis elaborados con la población que comete actos antisociales y delictivos desde la Teoría de la asociación diferencial propuesta por Sutherland (1947), en la que se explica que el comportamiento delictivo se aprende como cualquier otro y, además, tiene su origen en los procesos de interacción entre personas donde prima la comunicación, ya sea verbal o gestual, por lo que el foco de atención se ubica en los intercambios sociales (Matsueda, 2001). De este modo, identifica las variaciones que existen ya sea en la frecuencia, duración, prioridad e intensidad que acontecen durante las interacciones entre las personas, así como la edad, el prestigio de los agentes y el contexto donde se sucedan las mismas (Sutherland, 1947).

Akers (2006) habla de una Teoría de Aprendizaje Social, desde la cual plantea que existe una mayor probabilidad de que una persona cometa violaciones a la ley o actos desviados cuando: 1) se relaciona con personas que realiza, modelen, apoyen o propugnen definiciones favorables a estas; 2 ) a la conducta que va en contra de la norma le sigue una recompensa mayor al castigo que se puede recibir y, 3) cuando las definiciones propias, actitudes y creencias sean más favorables a la realización de actos contra la norma que la abstención de los mismos.

De esta manera, las propuestas actuales de reinserción social trazan una visión paradigmática, que se adhiera a un sistema de valores y restricciones, priorizando la obtención de ingresos económicos por la vía legítima sin verse en la necesidad de ejercer acciones ilegales o violentas por encima de las demás personas (Rumbo, 2013). El objetivo pues, no es homogeneizar bajo un molde de ciudadano bueno y ejemplar a toda la sociedad, sino que la postura y actuar del Estado y de sus instituciones se encaminen a contemplar las diferencias, la heterogeneidad de los proyectos de vida de cada persona, sus historias personales y características; todo lo anterior dentro de un marco de respeto al Derecho, sin que ello 
signifique violentar los derechos de las víctimas o del resto de la sociedad (González \& Morales, 2011).

Como se ha descrito, el significado que se consigue otorgar al término de reinserción social no se limita únicamente a la ausencia de conductas delictivas una vez egresando del sistema, es decir, a la reincidencia, sino que abarca aspectos clave como el respeto a la dignidad de la persona privada de su libertad y de las víctimas de los actos delictivos, permeando hasta la prevención, la efectiva procuración de justicia y la seguridad pública en la que la sociedad y el Estado sean partícipes. En razón a esta problemática, se decidió realizar un análisis de datos secundarios, empleando la literatura científica para identificar el significado y las características que según los estudiosos del tema conforman la reinserción social.

\section{Método}

Como primer paso, se efectuó una revisión de la legislación vigente en materia penal dirigida a personas adolescentes en conflicto con la ley en México. Se ubicaron dos niveles: legislación internacional y nacional. El tema en concreto en el que se enfocó la revisión fue identificar el concepto de reinserción social, así como el objetivo que se persigue con la implementación de la medida de privación de la libertad.

La finalidad de este ejercicio fue enmarcar legalmente las interpretaciones que se han realizado sobre el concepto de reinserción social en población adolescente. Así mismo, se trató de una introducción al marco normativo que, como se verá más adelante, tiene interrelación con las ideas de diversos autores que se desenvuelven en los ámbitos del derecho y la criminología, entre otros. En la siguiente tabla se refieren los hallazgos registrados. 
Tabla 1. Legislación vigente en México en materia de adolescentes en conflicto con la ley y el concepto de reinserción social

\begin{tabular}{|c|c|c|c|}
\hline Nivel & Legislación & Reinserción social & Privación de la libertad \\
\hline Internacional & $\begin{array}{l}\text { Reglas mínimas de las } \\
\text { Naciones Unidas para } \\
\text { la administración de la } \\
\text { justicia de menores }\end{array}$ & $\begin{array}{l}\text { Regla 26: Garantizar el } \\
\text { cuidado y Protección de } \\
\text { los menores, así como su } \\
\text { educación y formación } \\
\text { profesional. }\end{array}$ & $\begin{array}{l}\text { Regla 19.1: Se utilizará } \\
\text { como último recurso } \\
\text { y por el tiempo más } \\
\text { breve posible. }\end{array}$ \\
\hline Internacional & $\begin{array}{l}\text { Reglas de las Naciones } \\
\text { Unidas para la protec- } \\
\text { ción de los menores } \\
\text { privados de libertad }\end{array}$ & $\begin{array}{l}\text { Regla 12: Promover el sen- } \\
\text { tido de la responsabilidad e } \\
\text { impulsar actitudes y cono- } \\
\text { cimientos que ayuden a lo } \\
\text { menores a potenciar sus } \\
\text { capacidades como miem-- } \\
\text { bros de la sociedad. }\end{array}$ & $\begin{array}{l}\text { Regla 2: Será el último } \\
\text { recurso y durante el } \\
\text { tiempo mínimo nece- } \\
\text { sario. }\end{array}$ \\
\hline Nacional & $\begin{array}{l}\text { Constitución Política } \\
\text { de los Estados Unidos } \\
\text { Mexicanos }\end{array}$ & $\begin{array}{l}\text { Artículo 18: lograr la re- } \\
\text { inserción social y familiar } \\
\text { de la persona adolescente, } \\
\text { priorizando el pleno de- } \\
\text { sarrollo de su persona y } \\
\text { capacidades. }\end{array}$ & $\begin{array}{l}\text { Artículo 18: Se utiliza- } \\
\text { rá como medida ex- } \\
\text { trema y por el tiempo } \\
\text { más breve posible. }\end{array}$ \\
\hline Nacional & $\begin{array}{l}\text { Ley Nacional del Siste- } \\
\text { ma Integral de Justicia } \\
\text { Penal para Adoles- } \\
\text { centes }\end{array}$ & $\begin{array}{l}\text { Artículo 29: Restitución } \\
\text { del pleno ejercicio de los } \\
\text { derechos y libertades tras el } \\
\text { cumplimiento de las medi- } \\
\text { das ejecutadas. }\end{array}$ & $\begin{array}{l}\text { Artículo } 31 \text { : Es una } \\
\text { medida extrema, con } \\
\text { un tiempo determi- } \\
\text { nado y lo más breve } \\
\text { posible. }\end{array}$ \\
\hline
\end{tabular}

Fuente: elaboración propia.

Posteriormente, con el fin de complementar y profundizar en el tema, se realizó una revisión sistemática de literatura científica utilizando la herramienta de Google Académico en junio de 2017 con una muestra inicial de 93 artículos. Se seleccionó esta herramienta debido a que es un buscador que tiene la posibilidad de dar cuenta de los títulos, resúmenes y trabajos en extenso de la mayoría de las publicaciones actuales; también tiene la cualidad de ser un medio abierto a cualquier usuario sin necesidad de paga, es decir, brinda los elementos de acceso y conceptualización con mayor alcance para población académica, por lo tanto, aquel que será más difundido y socorrido. La revisión sistemática es un tipo de investigación que trabaja con datos secundarios (ya publicados) la cual "tiene como objetivo reunir toda la evidencia empírica que cumple unos criterios de elegibilidad previamente establecidos, 
con el fin de responder una pregunta específica de investigación. Utiliza métodos sistemáticos y explícitos, que se eligen con el fin de minimizar sesgos, aportando así resultados más fiables a partir de los cuales se puedan extraer conclusiones y tomar decisiones" (Higgins \& Green, 2011, p. 16). En esta se siguen diferentes pasos sistematizados, que inician con la definición clara de objetivos, metodología, selección de muestra documental, criterios de búsqueda, resultados y sus implicaciones. Sánchez-Meca (2010) añade que la revisión sistemática "tiene como objetivo reunir toda la evidencia empírica que cumple unos criterios de elegibilidad previamente establecidos, con el fin de responder una pregunta específica de investigación. Utiliza métodos sistemáticos y explícitos, que se eligen con el fin de minimizar sesgos, aportando así resultados más fiables a partir de los cuales se puedan extraer conclusiones y tomar decisiones" (p. 62).

En este caso, la pregunta de investigación buscaba ahondar en el manejo del concepto de reinserción social y readaptación social. Las palabras clave ingresadas en el metabuscador fueron las siguientes:

a) Reinserción social. Ya que es el término principal que se busca identificar.

b) Readaptación social. Es el término que algunas leyes utilizan de manera indistinta o como sinónimo de reinserción.

c) Menores infractores. Aunque en 2016 en México se comienza a utilizar el término de "adolescentes en conflicto con la ley", a efectos de este proceso de revisión se alude a menores infractores, ya que así se identificaba a la población conformada por personas menores de edad, sujetas a una medida de sanción como producto de la realización de algún acto tipificado como delito penal en el marco legal.

d) Adicciones. Ya que en investigaciones en las que se trabaja con personas drogodependientes se habla de reinserción social, se ha decidido utilizar este término como filtro para excluir.

Los criterios de inclusión y exclusión utilizados contemplaban a los textos en el idioma español, cuya fecha de publicación estuviera entre los años 2012 y 2017. Los tipos de fuentes usadas fueron tesis, artículos de revistas especializadas y libros, sin discriminar que fueran de corte cuantitativo y/o cualitativo, provenientes de los campos de estudio de 
la psicología, el derecho y la criminología. Los tipos de fuentes que no se tomaron en cuenta fueron trabajos escolares, programas curriculares académicos y guías de estudio, así como los textos que presentaban información repetida de otros trabajos.

Se descartó la revisión de documentos escritos en idiomas distintos del español, ya que el eje central de este trabajo parte de la visión de un sistema integral para personas adolescentes basándose en el respeto a los derechos humanos, por lo que aquellos conceptos procedentes de sistemas de justicia distintos a los de Estados hispanohablantes, no se consideraron adecuados para el análisis de este trabajo. Lo anterior visto como una oportunidad de reflexión sobre la transición del sistema de justicia que se está dando en México, retomando las experiencias de otros países con quienes se pueden contrastar procesos históricos y sociales.

Se usaron los estudios que particularmente:

- Presentaban una definición del concepto de reinserción social de personas sujetas a una medida de privación de la libertad.

- Presentaban una definición del concepto de reinserción social de personas menores de edad sujetas a una medida de privación de la libertad.

Por otra parte, se descartaron los estudios sobre:

- Reinserción social de personas que no están sujetas a una medida de privación de la libertad.

- Reinserción social de personas drogodependientes.

La revisión se realizó en cuatro momentos distintos, que a continuación se describen. En la prueba piloto se obtuvieron un total de 695 resultados; sin embargo, el uso de la palabra "adolescentes", disminuyó los resultados, ya que en el concepto "menores infractores" dentro de los marcos jurídicos, no suele hacerse distinción entre la población de niños o niñas y de adolescentes hombres y mujeres, por lo que en la segunda etapa se decidió eliminar ambas. En la segunda revisión, al quitar las palabras clave "menores infractores" y "adolescentes" se identificó que los 89 resultados 
obtenidos no proporcionaban información oportuna, esto debido a que los datos únicamente correspondían a la población de adultos.

Es entonces que se optó por utilizar la palabra clave "menores infractores", con el fin de que la búsqueda corresponda a la "reinserción social" de personas menores de edad, obteniendo un total de 93 resultados. Dentro de los documentos resultantes, tres de ellos se encontraban fuera del intervalo de tiempo usado como criterio de inclusión (2005, 2008 y 2011) y 14 documentos correspondientes a tesis, ya que no permitían acceso en su portal electrónico, por lo que se decidió realizar una segunda exploración con los mismos criterios días después, con el fin de no sesgar la búsqueda e intentar tener acceso a los documentos faltantes ya indicados.

\section{Resultados}

De la revisión sistemática se obtuvieron un total de 93 resultados, de los cuales desde el inicio se descartó el 6,45\% por no cumplir con los criterios de inclusión (repetición de artículo), uno de los documentos no permitió el acceso para consulta y uno de ellos presentaba el mismo contenido que otro texto. Dos tesis y dos libros se encontraban fuera del rango de tiempo establecido, pero fueron publicados en línea posteriormente.

Derivado de lo anterior, se trabajó con el 20,93\% de los resultados que cumplieron cabalmente los criterios de revisión, es decir, con un total de 18 documentos: 1 artículo de revista académica, 2 libros y 15 trabajos de tesis. La zona geográfica a la que corresponden las producciones son: México (38,8\%), Ecuador (22,22\%), El Salvador (11,11\%), Nicaragua (11,11\%), España (5,55\%), Chile (5,55\%) y Argentina (5,55\%). Así mismo, la producción sobre el tema corresponde al 16,66\% para 2016, seguido del 27,77\% para 2015, del 22,22\% para 2013 y 2012, y por último del 11,11\% para 2014. 
Del total de documentos, únicamente dos tocan el tema de la situación de personas adolescentes y la reinserción social; por su parte, los demás se disponían a describir los elementos que influyen en el proceso para que una persona logre la reinserción una vez que ha egresado de la institución de seguridad y/o haya cumplido con la medida que le fue impuesta. En una de las fuentes se ha encontrado puntualmente una definición particular para la población adolescente, ya que las demás tienden a transcribir o adecuar lo tratado en el concepto que se destina a la población adulta. En la tabla 2 se presentan los datos de identificación de los documentos consultados:

Tabla 2. Datos de identificación de los documentos consultados

\begin{tabular}{|c|c|c|c|c|}
\hline Autor & Título & Tipo & País & Año \\
\hline $\begin{array}{l}\text { Calderón, G. S., } \\
\text { Corvera, T. P. y } \\
\text { Mejía, A. D. }\end{array}$ & $\begin{array}{l}\text { La medida de internamiento como mecanis- } \\
\text { mo de reinserción social y desarrollo integral } \\
\text { para evitar la reincidencia delictiva en los } \\
\text { menores. }\end{array}$ & Tesis & El Salvador & 2016 \\
\hline Gutiérrez R., F. & $\begin{array}{l}\text { Modelo concesionado de prisiones federales } \\
\text { en México. }\end{array}$ & Tesis & México & 2016 \\
\hline Santiago L., E. & $\begin{array}{l}\text { Propuesta de un programa de reinserción } \\
\text { social para exinternos de un centro peniten- } \\
\text { ciario con el propósito de prevenir la reinci- } \\
\text { dencia delictiva. }\end{array}$ & Tesis & México & 2015 \\
\hline Sandoval, J. P. & $\begin{array}{l}\text { Argumentación jurídica sobre medidas de } \\
\text { protección para niños y niñas que incurran } \\
\text { en acciones penales para la protección del } \\
\text { derecho a la integridad personal. }\end{array}$ & Tesis & Ecuador & 2015 \\
\hline Carnevale, C. A. & $\begin{array}{l}\text { Antecedentes penales y reinserción laboral } \\
\text { en Argentina. }\end{array}$ & Tesis & Argentina & 2015 \\
\hline $\begin{array}{l}\text { Arévalo, K. y } \\
\text { González, V. E. }\end{array}$ & $\begin{array}{l}\text { Estado actual del derecho penitenciario en } \\
\text { Chile: bases para el establecimiento de una } \\
\text { reforma. }\end{array}$ & Tesis & Chile & 2015 \\
\hline Morocho, C. A. & $\begin{array}{l}\text { Los centros de internamiento para adoles- } \\
\text { centes infractores (CAI) de la ciudad de } \\
\text { Quito, incumplen con el derecho constitu- } \\
\text { cional a la rehabilitación social integral de los } \\
\text { menores internos. }\end{array}$ & Tesis & Ecuador & 2014 \\
\hline
\end{tabular}




\begin{tabular}{|c|c|c|c|c|}
\hline Autor & Título & Tipo & País & Año \\
\hline $\begin{array}{l}\text { Morales, E. y } \\
\text { Dávila, E. E. }\end{array}$ & $\begin{array}{l}\text { Análisis jurídico del principio de reinserción } \\
\text { familiar y social de los adolescentes priva- } \\
\text { dos de libertad, a través de la aplicación de } \\
\text { disposiciones administrativas-penitenciarias } \\
\text { (reglamentarias) en correspondencia con la } \\
\text { norma secundaria (Código de la Niñez y la } \\
\text { Adolescencia) que regulan la ejecución de } \\
\text { las sanciones penales en el Centro Penal de } \\
\text { Adolescentes de Tipitapa, durante el primer } \\
\text { semestre del año Dos Mil Catorce. }\end{array}$ & Tesis & Nicaragua & 2014 \\
\hline Llamedo E., V. & $\begin{array}{l}\text { La reinserción en los centros penitenciarios } \\
\text { españoles: una historia de vida para valorar } \\
\text { el paso de la teoría a la práctica. }\end{array}$ & Tesis & España & 2013 \\
\hline Peralta, Orfa E. & $\begin{array}{l}\text { Políticas que garantizan el respeto de los } \\
\text { derechos Humanos en el Centro de Reha- } \\
\text { bilitación Social de Mujeres de la ciudad de } \\
\text { Cuenca. }\end{array}$ & Tesis & Ecuador & 2013 \\
\hline Díaz, K. J. & $\begin{array}{l}\text { Incorpórese al código de la niñez y adoles- } \\
\text { cencia, un tratamiento eficaz para el interna- } \\
\text { miento de los adolescentes infractores en el } \\
\text { ecuador. }\end{array}$ & Tesis & Ecuador & 2013 \\
\hline Vega, J. F. & $\begin{array}{l}\text { Hacia la judicialización de la institución } \\
\text { abierta en el estado de Nuevo León. }\end{array}$ & Tesis & México & 2013 \\
\hline Melgar, E. & $\begin{array}{l}\text { El Sistema de Reinserción social aplicado en } \\
\text { el centro de readaptación de menores de } \\
\text { Tonacateteque en el periodo 2009-2010. }\end{array}$ & Tesis & El Salvador & 2012 \\
\hline $\begin{array}{l}\text { Alonso, M., Gar- } \\
\text { cía, N. R., Ramí- } \\
\text { rez, M., Ruiz, J. } \\
\text { L. y Segura, P. }\end{array}$ & $\begin{array}{l}\text { Aplicación del presupuesto del gobierno del } \\
\text { estado de Campeche. }\end{array}$ & Tesis & México & 2012 \\
\hline $\begin{array}{l}\text { Carazo, A. M. y } \\
\text { López, M. A. }\end{array}$ & $\begin{array}{l}\text { Análisis de la figura de la libertad condicional } \\
\text { en la legislación penal nicaragüense. }\end{array}$ & Tesis & Nicaragua & 2012 \\
\hline Sánchez, G., A. & $\begin{array}{l}\text { La transformación del sistema penitenciario } \\
\text { federal. Una visión de Estado. }\end{array}$ & Libro & México & 2012 \\
\hline $\begin{array}{l}\text { Santacruz, R., } \\
\text { Santillán H., E. y } \\
\text { Santacruz M., D. }\end{array}$ & $\begin{array}{l}\text { La ejecución de sentencias en el sistema } \\
\text { acusatorio. }\end{array}$ & Libro & México & 2015 \\
\hline De la Rosa, P. I. & $\begin{array}{l}\text { El escenario carcelario y las reformas a la } \\
\text { normatividad penitenciaria en el Estado de } \\
\text { San Luis Potosí. ¿Han sido suficientes ante la } \\
\text { crisis de los penales en la entidad? }\end{array}$ & Revista & México & 2016 \\
\hline
\end{tabular}

Fuente: elaboración propia 
A continuación, se referirán los conceptos obtenidos, los cuales han sido categorizados en relación con el empleo de la terminología, así como de manera cronológica, lo que permite identificar las ideas trabajadas respecto al concepto de "reinserción social" en los últimos años.

\section{Integración o reintegración a sociedad}

Alonso, García, Ramírez, Ruiz y Segura (2012) definen que la reinserción social ocurre cuando un individuo que vivía al margen de la sociedad se integra a ella. De igual forma, Carazo y López (2012) apuntan que el término reinserción se emplea para dar cuenta de la situación que acontece cuando se integra nuevamente a un individuo que por alguna razón se encontraba fuera de la sociedad o comunidad.

\section{Modificación de factores negativos}

Carazo y López (2012) recuperan lo expuesto por Gómez, Ruíz y Antonio (2004), para quienes, dentro de un sistema penal progresivo, la reinserción social acontece cuando a una persona se le han modificado los factores negativos de su personalidad y se le ha dotado de una formación general idónea.

Galindo (2012) considera que la reinserción social concretamente se orienta a un método que no pretende castigar, sino ayudar a que quien delinque deje los malos hábitos que le llevaron a estar en esa situación, promoviendo su transformación en una persona útil a la sociedad.

\section{Responsabilidad y reparación}

Melgar (2012) puntualiza la reintegración como la adaptación de una persona en la sociedad luego de haber estado al margen de ella y, de esta forma explica que se trata de una acción educativa compleja e integral, que busca ejecutar acciones de responsabilidad, reparación e integración social de las personas adolescentes, con el fin de limitar los efectos que el internamiento puede generar. En segundo lugar, la autora señala la importancia como agente de vinculación de las instituciones encargadas del cumplimiento de medidas y les encomienda la tarea de 
proporcionar educación, capacitación laboral y rehabilitación para que la persona adolescente pueda reincorporarse de manera útil a la sociedad al final del proceso de aprendizaje y vinculación social.

Vega (2013) reflexiona sobre la situación actual en la que se pretende reinsertar a la persona que ha sido privada de la libertad mediante una integración gradual y supervisada en el medio social externo, y reconoce que habrá que proporcionar a la persona elementos que le ayuden a superar su conflictiva personal y adecuar su adaptación socio-familiar.

Calderón, Corvera y Mejía (2016) refieren que la reinserción social y familiar está dirigida por dos exigencias básicas; primero, las penas no deberán ser de larga duración ya que, de ser así, la reintegración de la persona sería una ilusión y segundo, que a lo largo de la ejecución la persona pueda tener relación con el mundo exterior. En personas menores de edad, consideran que la readaptación al medio una vez que egresen de la institución se dará de manera distinta, ya que en el proceso de internamiento habrán adquirido habilidades. Para estos autores en particular, este es el primer paso para que la persona menor logre readaptarse a la sociedad y se desenvuelva en la misma más fácilmente.

\section{Recuperación del infractor}

Para Díaz (2013), la reinserción social consiste en que la persona que ha sido privada de la libertad recupere los vínculos con su familia, comunidad, trabajo, etc., de manera progresiva; esto por medio de estrategias que le permitan retornar a la vida en libertad. Una postura muy similar a la de Ojeda (2008), quien repara en que la reinserción social es volver a encauzar al delincuente dentro de la sociedad

\section{Bienestar de la sociedad}

Para Peralta (2013), la reinserción debe ser total, por lo que las autoridades no pueden poner en libertad a personas que, aun habiendo cumplido con su condena, no cuentan con las condiciones necesarias para ser miembros activos y sujetos de bien para la sociedad, por lo que desde su punto de vista debe primar el bien de la sociedad en general por encima del individual. 


\section{Fortalecer o mejorar a la persona}

Morocho (2014) indica que es importante que exista una intervención de calidad y seguimiento de los logros readaptativos de las personas a fin de evitar la reincidencia. Para el autor, esto se alcanza cuando se fortalecen las capacidades personales, familiares y sociales, siempre que se tienda a la integración social y se desestimen los comportamientos delictivos.

En el caso de personas adolescentes en conflicto con la ley, para Morales y Dávila (2014) la demostración del efectivo respeto del interés superior del adolescente en la etapa de ejecución de las medidas penales, tendrá como resultado el cumplimiento del principio de reinserción social. Así mismo, suponen que este último se desarrolla en dos ámbitos, el familiar y el comunitario; la finalidad de las medidas impuestas corresponde a la transformación de la persona adolescente y su rehabilitación, por lo que se busca que el adolescente asuma papeles constructivos y útiles dentro de la sociedad y no reincida.

Siguiendo esta línea, Arévalo y González (2015) recuperan la definición que proporciona Spittel (2013), en la cual explica que contribuir a la reinserción social alude a la idea de ayudar a una persona y resalta la importancia de que la persona que salga de la cárcel debe estar preparada. Así como Carnevale (2015) coincide y expresa que la reinserción implicaría disponer de medios de tratamiento jurídicos y sociales para que las personas tengan una vida digna en el exterior, además de intentar minimizar la falta de socialización que de manera paralela en prisión ocurre.

Por su parte, Sandoval (2015), congruente con la legislación en Ecuador, menciona que las medidas aplicadas en menores de edad tienen un sentido protector bilateral, es decir, se asegura de resarcir el daño causado por la persona adolescente y se pretende salvaguardar el interés superior del menor, y ya que las sanciones que les corresponderían son medidas socioeducativas, se procura que cuando lleguen a la mayoría de edad logren entender la falta que cometieron y que en el futuro sean personas positivas para la sociedad. 
Santiago (2015) establece que la reinserción social es el proceso por el cual las personas que se encontraban privadas de la libertad pasan de estar detenidos a vivir en la comunidad, el cual se construye a partir de los factores internos y externos que coadyuven en la prevención de la reincidencia delictiva. Así mismo, recupera la definición propuesta por Espinoza (2008), para quien el proceso de la reinserción postcarcelaria consiste en re-institucionalizar a la persona en áreas clave como el trabajo, la familia y la planificación personal, entre otras.

En el caso mexicano, De la Rosa (2016) señala que, en conformidad con la reforma en materia de Derechos Humanos, durante el año 2011 en México, el numeral 18 de la Constitución estipula que los medios para lograr la reinserción de los sentenciados serán el trabajo, la capacitación, la educación, la salud y el deporte.

Desde el punto de vista de Gutiérrez (2016), son dos los pilares de la reinserción social: la clasificación y el tratamiento de internos. Por clasificación se refiere a la investigación y evaluación de las personas privadas de la libertad, labor realizada por especialistas; para el tratamiento expone la importancia que tiene el seguimiento y monitoreo del avance del interno en términos de reinserción. Por lo tanto, la meta de los programas de reinserción social es apoyar al delincuente para que abandone la realización de actividades delictivas, es decir, que no vuelva a cometer más delitos (Gutiérrez, 2016).

En general, la postura inicial de los autores revisados se concentra en la propuesta de no reincidencia y la prevención de futuras conductas delictivas por parte del sentenciado; además, es posible identificar algunas diferencias significativas en cuanto a los elementos clave que consideran como punto de partida de la reinserción social, ampliando así las áreas que pueden ser trabajadas de forma cooperativa entre especialistas del sistema penal, las cuales, a pesar de estar enmarcadas por la disciplina desde la que fueron configurados y a razón del objeto de estudio en la que se concentraron los diversos autores que se consultaron en esta revisión, permiten vislumbrar el horizonte hacia el que se encamina la reinserción social en los últimos años. 
Es así que, en un primer momento se distingue la idea radical o dual en la que a la persona en reclusión se le encuadra bajo un marco jurídico-social, es decir, la condición en la que en un momento se encuentra aislado de la sociedad y posteriormente se integra a ella, por lo que esta base se restringe simplemente a la duración de la sentencia indicada por el juez hasta su culminación. Dentro de esta propuesta, se destaca una más rigurosa que establece que si el individuo en cuestión no se encuentra listo, aunque su sentencia esté concluida, no deberá de ser puesto en libertad.

En segundo lugar, los autores señalan la conveniencia de transformar a la persona sentenciada en alguien útil a la sociedad, lo cual se guía bajo dos premisas principales. La primera correspondería a la responsabilidad que recae por completo en el individuo que se supone será integrado a la sociedad nuevamente, siempre y cuando logre modificar elementos negativos de sí mismo, a fin de estar preparado para el momento en que salga de la institución donde se encuentra.

La segunda se refiere a la responsabilidad compartida que existe entre las instituciones involucradas en la impartición de justicia y la sociedad en general, destacando la participación activa de grupos como la familia y la comunidad a la que pertenece el individuo, propiciando su desarrollo bajo intervenciones de calidad en áreas como la educación, la capacitación laboral, entre otros. En la tabla 3 se sintetizan las ideas de los autores, mismas que en el apartado de conclusiones serán discutidas a mayor profundidad.

Tabla 3. Esferas de la reinserción social derivadas de los resultados obtenidos en la revisión sistemática de información

\begin{tabular}{|l|l|l|}
\hline \multicolumn{2}{|c|}{ Reinserción social: No reincidencia y prevención de delitos futuros } \\
\hline \multirow{3}{*}{ Situación jurídica-social } & Postura radical o dual. & $\begin{array}{l}\text { Aunque el individuo cumpla con su } \\
\text { sentencia, si no está listo no debe de ser } \\
\text { puesto en libertad. }\end{array}$ \\
\cline { 3 - 3 } & $\begin{array}{l}\text { Momento en que un individuo alejado de } \\
\text { la sociedad se reintegra a ella. }\end{array}$ \\
\hline
\end{tabular}

Continúa 


\begin{tabular}{|c|c|c|}
\hline \multicolumn{3}{|c|}{ Reinserción social: No reincidencia y prevención de delitos futuros } \\
\hline \multirow{6}{*}{$\begin{array}{l}\text { Convertir a la perso- } \\
\text { na en alguien útil a la } \\
\text { sociedad }\end{array}$} & \multirow{3}{*}{$\begin{array}{l}\text { Responsabilidad centra- } \\
\text { da en el individuo. }\end{array}$} & $\begin{array}{l}\text { Modificación de aspectos negativos de la } \\
\text { personalidad. }\end{array}$ \\
\hline & & $\begin{array}{l}\text { Ayudar a que el individuo deje sus malos } \\
\text { hábitos. }\end{array}$ \\
\hline & & $\begin{array}{l}\text { Preparación previa al momento de regre- } \\
\text { sar al exterior. }\end{array}$ \\
\hline & \multirow{3}{*}{$\begin{array}{l}\text { Responsabilidad com- } \\
\text { partida entre las institu- } \\
\text { ciones involucradas en } \\
\text { la impartición de justicia } \\
\text { y la sociedad en general. }\end{array}$} & $\begin{array}{l}\text { Proporcionar educación, capacitación } \\
\text { laboral y rehabilitación. }\end{array}$ \\
\hline & & $\begin{array}{l}\text { Recuperar vínculos con la familia y co- } \\
\text { munidad. }\end{array}$ \\
\hline & & Tratamientos e intervenciones de calidad. \\
\hline
\end{tabular}

Fuente: elaboración propia.

\section{Conclusión}

La reinserción social es la incorporación de una persona a la sociedad de la que se entraba al margen (Alonso et al., 2012; Carazo \& López, 2012; Santiago, 2015). En este sentido, se desprenden dos líneas de análisis que, si bien no son excluyentes, generan una diferencia en la forma de pensar sobre las personas que se encuentran reclusas. Primero, la reincidencia como un indicador categórico y la conveniencia de la participación activa de la persona privada de la libertad con el exterior.

En segundo lugar, la no reincidencia se identifica como un elemento asociado al éxito de la intervención realizada durante la estancia en los centros de seguridad (Flores, s.f.; Gutiérrez, 2016); sin embargo, cabe la posibilidad de que quien cometa un acto delictivo nuevamente no llegue a ser denunciado o que cometa la acción en un lugar distinto a aquel en donde previamente se le procesó, razón por la cual es pertinente indagar a partir de indicadores de otra índole. Por ejemplo, analizar el sistema de valores que impera en los espacios de los que procede el sentenciado y al que deberá regresar cuando salga de la institución de seguridad, pues la probabilidad de que se realicen violaciones a la ley aumenta cuando: a) la interacción social ocurre dentro de un contexto en el cual imperan definiciones favorables a dichas conductas, b) las recompensas pueden 
ser mayores que el castigo o c) cuando las definiciones propias favorecen la realización más que a la abstención, por lo tanto, sería complicado que una persona no vuelva a delinquir si se encuentra en un el lugar que promueve lo contario (Akers, 2006; Baratta, 2004).

La participación activa de la persona privada de la libertad con el exterior (Díaz, 2013; Morocho, 2014; Calderón, et al., 2016) y el papel primordial que tienen los grupos como la familia y la comunidad en la que se incorporará una vez que recupere su libertad es de suma importancia; este aspecto concuerda con lo establecido en instrumentos internacionales como las Reglas de la Habana, los Principios básicos para el tratamiento de reclusos y la Convención de los Derechos del Niño. A pesar de que este es un factor clave para promover conductas que vayan en beneficio de la socialización de quien se encuentra dentro de una institución de seguridad, en el caso de México, los centros de detención para adolescentes se encuentran en las zonas más céntricas de los estados, por lo que la mayoría de personas que se encuentran en ellas están apartados de sus lugares de origen, ${ }^{2}$ disminuyendo con esto la posibilidad de interactuar con sus grupos sociales primarios.

En caso de que el contacto con el exterior se dé en las mejores condiciones posibles, podría lograrse eventualmente la restructuración en los esquemas de interacción en cuanto a frecuencia, duración e intensidad de la persona que ha cometido un acto delictivo, orientándose así hacia la abstención de este tipo de conductas (Matsueda, 2001).

Otro elemento de gran relevancia en estas investigaciones es la incorporación gradual de las personas detenidas a la sociedad mediante una preparación previa (Vega, 2013; Arévalo \& González, 2015). Destacando las actividades orientadas hacia la educación, la capacitación, el trabajo y la participación comunitaria (Santacruz et al., 2015; De la Rosa, 2016), coincidiendo con lo plasmado en la CPEUM y los cinco ejes que considera para la reinserción social.

2 Hasta el año 2016, la infraestructura penitenciaria para los 32 estados que conforman México, mantenía en operación 55 centros destinados a la atención de población adolescente. Exceptuando los casos de la Ciudad de México y Sonora los cuales contaban con 6 centros; Tamaulipas con 5; Baja California con 3 y Coahuila de Zaragoza, Chiapas, Chihuahua, Durango, Jalisco y Nuevo León con 2 respectivamente. Ver Censo Nacional de Gobierno, Seguridad Pública y Sistema Penitenciario Estatales. "Recursos en los centros de tratamiento para adolescentes" (2018). 
Sobre las intervenciones en el ámbito tanto jurídico como social, el énfasis se centra en la aportación de los tratamientos de calidad (Morocho, 2014; Carnevale, 2015), aunque no se detallan cuáles serían los procedimientos que sí se consideran indispensables para que quien se encuentra privado de la libertad pueda adaptarse a la sociedad en el exterior. En este sentido, hasta el momento el abordaje cognitivo conductual ha sido el que mayores aportes y beneficios ha traído al sistema penitenciario (Sánchez-Armáss \& García, 2012), pese a que en México aún no existen estudios que permitan identificar los programas con mayor impacto en la población de adolescentes con una medida de privación de la libertad en particular.

Peralta (2013) expresa que, a pesar de haber cumplido con la sanción impuesta, es mejor que no egresen las personas de los centros de detención de no contar con lo necesario para mantener el bienestar social, contraponiéndose así a la propuesta de Calderón et al., (2016) quienes consideran que las penas debieran ser de corta duración.

Para Gómez et al. (2004), la intervención del sistema penitenciario se debe orientar a la modificación de los aspectos negativos de cada individuo; sin embargo, la responsabilidad de un cambio sobre sí mismo, sin la participación activa de todos los agentes posibles y más aún, sin establecer parámetros sobre los cuales comparar los efectos que la estancia en una institución de este tipo tiene en la vida de las personas y las consecuencias que de ello derive una vez que se encuentren en el exterior, puede afectar los esfuerzos que se realizan desde diferentes ámbitos del sistema de justicia. Por ejemplo, el cambio legislativo que ocurrió en los últimos años en México en materia penal se ve ensombrecido por las violaciones a los Derechos Humanos que ocurren dentro de los centros de detención (Comisión Nacional de Derechos Humanos, 2018), así como el trabajo realizado desde la sociedad civil, en donde los esfuerzos se encaminan principalmente a colaborar como una red de apoyo externa que busca satisfacer las necesidades que el Estado no alcanza a cubrir. ${ }^{3}$ 
Para la población adolescente se vislumbran tres puntos clave que se distinguen de lo referido para la adulta: primero, se pretende que logren ser personas productivas en la sociedad o que contribuyan con su comunidad (Morales \& Dávila, 2014; Sandoval, 2015), lo cual implica la factibilidad de un cambio positivo en su actuar. En segundo lugar, se destaca el interés superior del menor, el cual refiere que por encontrarse en un momento de desarrollo y crecimiento trascendental, es indispensable una intervención que amplíe sus capacidades, aminorando las consecuencias del castigo, es decir, el encarcelamiento, el cual, debido a su naturaleza, es aversivo; en tercer lugar, se propone que la reinserción social es un proceso de aprendizaje y vinculación social, tomando en cuenta la reparación del daño a la víctima y promoviendo acciones donde cada adolescente logre asumir la responsabilidad de su actuar (Melgar, 2012).

El papel de la sociedad y el Estado se establece en el marco de la satisfacción de los derechos básicos de los que cada persona menor de edad es tutelar, a fin de que existan opciones y alternativas de estilos de vida entre las que puedan elegir (Rumbo, 2013; Valenzuela, 2015). En este sentido, se puede retomar a Becker (1963) y su teoría del etiquetamiento social, ya que se criminaliza la imagen de la persona joven que cumple con ciertas características asociadas a conceptos negativos (Valenzuela, 2015; di Napoli, 2016), instaurando así un estigma social sobre la persona adolescente, a pesar de haber cumplido legalmente con la medida que correspondía a su conducta, por lo que sería conveniente la intervención y participación de los grupos más cercanos e íntimos como la familia, su grupo de pares y la comunidad, a fin de evitar la reincidencia y que su integración efectiva no se vea comprometida.

Para la población adolescente, al menos en México, el máximo tiempo de privación de libertad corresponde a cinco años, ${ }^{4}$ por lo cual es oportuno analizar en qué condiciones las personas jóvenes regresan a sus espacios de origen mediante un seguimiento adecuado. Es así que el

4 Es conveniente recordar que, como anteriormente se indicó, el art. 145 de la LNSIJPA señala que la duración máxima de la medida de internamiento será de tres años para adolescentes de 14 años cumplidos a menos de 16 y una máxima de cinco años en casos de 16 años cumplidos a menos 18. Además, indica también que la duración máxima del internamiento podrá ser de hasta cinco años en los casos de homicidio calificado, violación tumultuaria, secuestro; hechos señalados como delitos en materia de trata de personas y delincuencia organizada. 
término reinserción social no debe de obedecer únicamente a un sentido de armonización de legislaciones internacionales, federales y locales, sino que debe de promover el cambio de paradigma tanto en operadores del sistema en su actuación durante los procesos penales y la correcta aplicación de la ley, así como de la sociedad en general, buscando así que se involucren y sean responsables de sus acciones y omisiones en el tema de adolescentes en conflicto con la ley.

En cuanto al tema de implementación de programa e intervenciones desde varios campos del saber, sería conveniente que se establezcan, a partir de un plan de trabajo estructurado con objetivos e indicadores claros, parámetros que permitan monitorear avances o retrocesos. Otro elemento que sobresale en los resultados obtenidos en esta revisión es que, en cada una de las definiciones proporcionadas por las y los autores, no se hace alusión a la percepción de las y los adolescentes, sus necesidades o los contextos a los que pertenecen; de aquellos que han egresado de los centros de detención y las experiencias que sirvieron o fueron ineficaces a su vuelta a la comunidad o aquellas que suscitaron la reincidencia, elemento clave a tener en cuenta para posteriores revisiones e investigaciones.

\section{Referencias}

Aguilar, R. \& Castañeda, J. (2012). La guerra antinarco el gran fracaso de Calderón. Recuperado de https://www.proceso.com.mx/322831/la-guerra-antinarcoel-gran-fracaso-de-calderon.

Akers, R. L. (2006). Aplicaciones de los principios del aprendizaje social. Algunos programas de tratamiento y prevención de la delincuencia. En Bueno, F., Guzmán, J. L., \& Serrano, A. (coords.), Derecho penal y criminología como fundamento de la política criminal: estudios en homenaje al profesor Alfonso Serrano Gómez (pp. 1117-1138). Madrid: Dykinson.

Alonso, M., García, N. R., Ramírez, M., Ruiz, J. L., \& Segura, P., (2012). Aplicación del presupuesto del gobierno del estado de Campeche. (Tesis de licenciatura), Instituto Politécnico Nacional, México. Recuperado de https://tesis. ipn.mx/xmlui/bitstream/handle/123456789/13474/CP2012\%20A456m. pdf?sequence $=1$ GisAllowed $=y$ 
Arévalo, K. E González, V. E. (2015). Estado actual del derecho penitenciario en Chile: bases para el establecimiento de una reforma. (Tesis de licenciatura), Universidad de Chile, Chile. Recuperado de http://repositorio.uchile.cl/ handle/2250/130682

Asamblea General de las Naciones Unidas. Convención sobre los Derechos del Niño, resolución 44/25, 20-11-1989. Recuperado de https://www.ohchr.org/SP/ Professionallnterest/Pages/CRC.aspx

Asamblea General de las Naciones Unidas. Pacto Internacional de Derechos Civiles y Políticos, resolución 2000 A (XII), 6-12-1966. Recuperado de https://www. ohchr.org/SP/Professionallnterest/Pages/CCPR.aspx

Asamblea General de las Naciones Unidas. Principios básicos para el tratamiento de los reclusos, resolución 45/111, 14-12-1990. Recuperado de https://www.ohchr. org/SP/Professionallnterest/Pages/BasicPrinciplesTreatmentOfPrisoners.aspx Asamblea General de las Naciones Unidas. Reglas de las Naciones Unidas para la protección de los menores privados de libertad (Reglas de la Habana), resolución 45/113, 14-12-1990. Recuperado de http://www.ordenjuridico.gob.mx/ TratInt/Derechos\%20Humanos/OTROS\%2019.pdf

Asamblea General de las Naciones Unidas. Reglas minimas de las Naciones Unidas para la administración de la justicia de menores (Reglas de Beijing), resolución 40/33, 29-11-1985. Recuperado de http://www.ordenjuridico.gob.mx/ TratInt/Derechos\%20Humanos/OTROS\%2018.pdf

Baratta, A., (2004). Criminología y sistema penal. Buenos Aires: B de F.

Becker, H. S. (1963). Outsiders: Studies in the Sociology of Deviance. Nueva York: The Free Press of Glencoe.

Calderón, G. S., Corvera, T. P., E Mejía, A. D. (2016). La medida de internamiento como mecanismo de reinserción social y desarrollo integral para evitar la reincidencia delictiva en los menores. (Tesis de licenciatura), Universidad de El Salvador, El Salvador. Recuperado de http://ri.ues.edu.sv/11410/

Carazo, A. M. E López, M. A. (2012). Análisis de la figura de la libertad condicional en la legislación penal nicaragüense (Tesis de licenciatura), Universidad Centroamericana, Nicaragua. Recuperado de http://repositorio.uca.edu.ni/412/ Carnevale, C. A. (2015). Análisis de la figura de la libertad condicional en la legislación penal nicaragüense (Tesis de Maestría). Universidad Nacional del Sur, Argentina. Recuperado de http://repositoriodigital.uns.edu.ar/bitstream/123456789/2876/1/TESIS\%20CARNEVALE.pdf

Comisión Nacional de Derechos Humanos. (2018). Informe de actividades, 2018. Personas privadas de la libertad. Recuperado en http://informe.cndh.org.mx/ menu.aspx? $\mathrm{id}=30087$ 
Daniel, J. E Zepeda, R. (2015). La guerra contra el narcotráfico en México: una guerra perdida. Reflexiones, (94), 153-168.

De la Rosa, P. I. (2016). El escenario carcelario y las reformas a la normatividad penitenciaria en el Estado de San Luis Potosí. ¿Han sido suficientes ante la crisis de los penales en la entidad? Ciencia Jurídica, 5(9), 7-25.

Decreto que se reforman y adicionan diversas disposiciones de la Constitución Política de los Estados Unidos Mexicanos, Diario Oficial de la Federación [DOF], 18-06-2008, (Méx.). Recuperado de http://dof.gob.mx/nota_detalle. php?codigo $=5046978$ \&fecha $=18 / 06 / 2008$

Di Napoli, P. (2016). La juventud como objeto de temor y estigma. Sentimientos desde y hacia los jóvenes de los países del Cono Sur. Revista de Ciencias Sociales, 29(38), 123-144.

Díaz, K. J. (2013). Incorpórese al código de la niñez y adolescencia, un tratamiento eficaz para el internamiento de los adolescentes infractores en el ecuador. (Tesis de Licenciatura), Universidad Nacional de Loja, Ecuador. Recuperado de http://dspace.unl.edu.ec/handle/123456789/6739

Espinosa L., I. P. \& Zaragoza, H., J. (2017). Criminología Penitenciaria. De la regeneración a la restauración: cien años de penitenciarismo mexicano. Archivos de Criminología, Seguridad Privada y Criminalística, 5(19), 56-68.

Santiago Lorezana, E. (2015). Propuesta de un programa de reinserción social para ex internos de un centro penitenciario con el propósito de prevenir la reincidencia delictiva. (Tesis de Licenciatura. Universidad Autónoma del Estado de México, México). Recuperado de http://ri.uaemex.mx/handle/20.500.11799/62614

Flores, B. (s.f.). Fundamento del nuevo sistema penitenciario y el juez ejecutor de sentencias. Recuperado de http://congresopuebla.gob.mx/docs/Mesa2/35_FUNDAMENTO_DEL_NUEVO_SISTEMA_PENITENCIARIO_Y_EL_JUEZ_EJECUTOR_DE_SENTENCIAS.pdf

Carazo Rodríguez, A. M. E López Moreno, M. A. (2012). Análisis de la figura de la libertad condicional en la legislación penal nicaragüense. (Tesis de licenciatura. Universidad Centroamericana, Nicaragua). Recuperado de http://repositorio.uca.edu.ni/412/

González, P., L. E Morales S., J. (2011). Los derechos humanos de las personas privadas de la libertad: fundamento de la ejecución de la pena y la reinserción social. En F. G. Campos Domínguez, D. Cienfuegos Salgado, L. G. Rodríguez Lozano, \& J. Zaragoza Huerta (coords.) Entre libertad y castigo: dilemas del estado contemporáneo. Estudios en homenaje a la Maestra Emma Mendoza Bremauntz (pp. 373-381). México: Editorial Laguna. 
Gutiérrez R., F. (2016). Modelo concesionado de prisiones federales en México, (Tesis de Maestría), Instituto Tecnológico y de Estudios Superiores de Monterrey, México. Recuperado de https://repositorio.itesm.mx/handle/11285/619707 Higgins, J. P. \& Green, S. (2011). Manual Cochrane de revisiones sistemáticas de intervenciones. Recuperado de https://es.cochrane.org/sites/es.cochrane.org/ files/public/uploads/Manual_Cochrane_510_reduit.pdf

Instituto Nacional de Estadística y Geografía. (2017). Censo Nacional de Gobierno, Seguridad Pública y Sistema Penitenciario Estatales. Recuperado de http://www.beta.inegi.org.mx/programas/cngspspe/2017/

Instituto Nacional de Estadística y Geografía. (2018). Censo Nacional de Gobierno, Seguridad Pública y Sistema Penitenciario Estatales. "Recursos en los centros de tratamiento para adolescentes". Recuperado de https://www.inegi.org.mx/ sistemas/olap/consulta/general_ver4/MDXQueryDatos.asp?\#Regreso\& $\mathrm{c}=$ Instituto Nacional de Estadística y Geografía. (2018). Encuesta Nacional de Victimización y Percepción sobre Seguridad Pública. Recuperado de http://www. beta.inegi.org.mx/proyectos/enchogares/regulares/envipe/2018/default.html

Ley Nacional del Sistema Integral de Justicia Penal para Adolescentes. Diario Oficial de la Federación, [DOF]. 16-06-2016, (Méx.). Recuperado de http://dof.gob.mx/nota_to_imagen_fs.php?cod_diario=270361 $\mathrm{Gpagina}=-$ 1 \&fecha $=16 / 06 / 2016$

Llamedo E., V. (2013). La reinserción en los centros penitenciarios españoles: una historia de vida para valorar el paso de la teoría a la práctica. (Trabajo de Grado en Educación Social. Universidad de Valladolid, España). Recuperado de http://uvadoc.uva.es/handle/10324/4247

Matsueda, R. L., (2001), Differential association theory. En C. D. Bryant (Ed.), Historical, conceptual, and Theoretical Issues (pp. 125-130). Philadelphia: Brunner-Routledge.

Melgar, E. (2012). El Sistema de Reinserción social aplicado en el centro de readaptación de menores de Tonacateteque en el periodo 2009-2010. (Tesis de Licenciatura. Universidad de El Salvador, El salvador). Recuperado de http://ri.ues.edu. sv/2846/

Mendoza, K. (2011). Aspectos básicos para comprender la reforma constitucional en materia de justicia penal. Programa de capacitación y formación profesional en derechos humanos. Fase de actualización permanente. México: Comisión de Derechos Humanos del Distrito Federal. Recuperado de http://cdhdf.org. mx/serv_prof/pdf/asp_basicos.pdf 
Molzahn, C., Rodríguez, O., E Shirk, D. A. (2013). Drug violence in Mexico: Data and analysis through 2012. San Diego: Trans-Border Institute, University of California.

Morales, E. E Dávila, E. E. (2014). Análisis jurídico del principio de reinserción familiar y social de los adolescentes privados de libertad, a través de la aplicación de disposiciones administrativas-penitenciarias (reglamentarias) en correspondencia con la norma secundaria (Código de la Niñez y la Adolescencia) que regulan la ejecución de las sanciones penales en el Centro Penal de Adolescentes de Tipitapa, durante el primer semestre del año Dos Mil Catorce, (Tesis de Licenciatura), Universidad Nacional Autónoma de Nicaragua, Nicaragua). Recuperado de http://repositorio.cnu.edu.ni/Record/RepoUNANM520

Morocho, C. A. (2014). Los centros de internamiento para adolescentes infractores (CAI) de la ciudad de Quito, incumplen con el derecho constitucional a la rehabilitación social integral de los menores internos. (Tesis de Licenciatura), Universidad Central del Ecuador, Ecuador. Recuperado de http://www.dspace.uce.edu. ec/handle/25000/3274

Ojeda, J. (2012). Reinserción social y función de la pena. Recuperado de https:// archivos.juridicas.unam.mx/www/bjv/libros/7/3169/7.pdf

Peralta, Orfa E. (2013). Políticas que garantizan el respeto de los derechos Humanos en el Centro de Rehabilitación Social de Mujeres de la ciudad de Cuenca. (Tesis de Licenciatura) Universidad del Azuay, Ecuador. Recuperado de http:// dspace.uazuay.edu.ec/handle/datos/472

Pérez, J. A. (2011). La explicación sociológica de la criminalidad. Derecho y cambio social, 22(7), 1-22.

Red por los Derechos de la Infancia en México (2011). Infancia y conflicto armado en México. Informe alternativo sobre protocolo facultativo de la Convención sobre los derechos del niño, relativo a la participación de niños en los conflictos armados. Recuperado de http://derechosinfancia.org.mx/ documentos/iaespanol.pdf

Rosen, J. E Zepeda M., R. (2015). La guerra contra el narcotráfico en México: una guerra perdida. Reflexiones, 94(1), 153-168. Recuperado de https:// www.redalyc.org/pdf/729/72941346011.pdf

Rumbo, C. (2013), Los retos del proceso de reinserción social en el Estado mexicano, frente a la figura del penitenciarismo en Colombia. Revista Logos, ciencia y Sánchez, G., A. (coord.) (2012). La transformación del Sistema Penitenciario Federal: una visión de estado. México: SSP, CIES.

Sánchez-Armáss C. O. E García de Alba V. J. (2012). Readaptación social de menores de edad: una revisión de estudios empíricos. Revista electrónica de 
Psicología Iztacala, 15(4), 1412-1439. Recuperado de http://www.revistas. unam.mx/index.php/repi/article/view/34788

Sánchez-Meca, J. (2010). Cómo realizar una revisión sistemática y un metaanálisis. Aula aberta, 38(2), 53-64.

Sandoval, J. P. (2015). Argumentación jurídica sobre medidas de protección para niños y niñas que incurran en acciones penales para la protección del derecho a la integridad personal. (Tesis de Licenciatura), Universidad Regional Autónoma de los Andes, Ecuador. Recuperado de http://186.3.45.37/handle/123456789/1425.

Santacruz Fernández, R., Santillán Huerta, E. E Santacruz Morales, D. (2015). La ejecución de sentencias en el sistema acusatorio. México: Benemérita Universidad Autónoma de Puebla. Recuperado de http://cmas.siu.buap.mx/portal_pprd/ work/sites/fdcs/resources/PDFContent/1389/Libro\%20DIG\%20-\%20 Ejecuci\%C3\%B3n\%20de\%20sentencias.pdf

Santacruz, R., Santillán H., E., E Santacruz M., D. (2015). La ejecución de sentencias en el sistema acusatorio. México: Benemérita Universidad Autónoma de Puebla. Recuperado de http://cmas.siu.buap.mx/portal_pprd/work/sites/fdcs/ resources/PDFContent/1389/Libro\%20DIG\%20-020Ejecuci\%C3\%B3n\%20 de $\% 20$ sentencias.pdf

Santiago, E. (2015). Propuesta de un programa de reinserción social para ex internos de un centro penitenciario con el propósito de prevenir la reincidencia delictiva. (Tesis de Licenciatura), Universidad Autónoma del Estado de México, México. Recuperado de http://ri.uaemex.mx/handle/20.500.11799/62614

Arévalo, K. E González. V. E. (2015). Estado actual del derecho penitenciario en Chile: bases para el establecimiento de una reforma. (Tesis de licenciatura. Universidad de Chile, Chile). Recuperado de http://repositorio.uchile.cl/ handle/2250/130682

Sutherland, E. H. (1947). Principles of Criminology (4 edición). EUA: J. B. Lippincott Company.

Valenzuela, J. M. (2015). Prólogo: Aunque nos sangre el corazón. En J. M. Valenzuela (Coord.), Juvenicidio: Ayotzinapa y las vidas precarias en América Latina y España (pp. 11-14). Barcelona: Nuevos emprendimientos editoriales.

Valenzuela, J. M. (2015). Remolinos de viento: juvenicidio e identidades desacreditadas. En J. M. Valenzuela Arce (Coord.), Ayotzinapa y las vidas precarias en América Latina y España (pp. 15-57). Barcelona: Ned Ediciones.

Vega, J. F. (2013). Hacia la judicialización de la institución abierta en el estado de Nuevo León. (Tesis de Doctorado), Universidad de Nuevo León, México. Recuperado de http://eprints.uanl.mx/11005/ 
Villagra, C. (2015). Hacia una política postpenitenciaria en Chile: desafíos para la reintegración de quienes salen de la cárcel. Revista Electrónica Debates Penitenciarios, 7(2), 2-14. Recuperado de https://www.cesc.uchile.cl/pub_periodicas_dp_07.htm

Zaffaroni, E. R. (1995). Los objetivos del sistema penitenciario y las normas constitucionales. En J. B. J. Maier y A. Binder (comps.), El derecho penal hoy. Homenaje al profesor David Baigún (pp. 118-120). Argentina: Editores del Peusto S. R. L. 